\title{
Prevalence and Spectrum of BRCA Germline Variants in Central Italian High Risk or Familial Breast/Ovarian Cancer Patients: A Monocentric Study
}

\author{
Jennifer Foglietta ${ }^{1,+}$, Vienna Ludovini ${ }^{2, *}+{ }^{+}$(D) Fortunato Bianconi ${ }^{3}$, Lorenza Pistola ${ }^{2}$, \\ Maria Sole Reda ${ }^{2}$, Antonella Al-Refaie ${ }^{2}$, Francesca Romana Tofanetti ${ }^{2}$, Annamaria Mosconi ${ }^{2}$, \\ Elisa Minenza ${ }^{2}$, Paola Anastasi ${ }^{2}$, Carmen Molica ${ }^{2}$, Fabrizio Stracci ${ }^{4}$ and Fausto Roila ${ }^{2}$ \\ 1 Medical Oncology Division, S. Maria Hospital, 05100 Terni, Italy; j.foglietta@aospterni.it \\ 2 Medical Oncology Division, S. Maria della Misericordia Hospital, 06132 Perugia, Italy; \\ lorenza.pistola@ospedale.perugia.it (L.P.); mariasolereda@inwind.it (M.S.R.); a_anto92@hotmail.it (A.A.-R.); \\ francesca.tofanetti@ospedale.perugia.it (F.R.T.); annamaria.mosconi@ospedale.perugia.it (A.M.); \\ elisa.minenza@ospedale.perugia.it (E.M.); paola.anastasi@ospedale.perugia.it (P.A.); \\ carmen.molica@ospedale.perugia.it (C.M.); fausto.roila@ospedale.perugia.it (F.R.) \\ 3 Umbria Cancer Registry, University of Perugia, 06129 Perugia, Italy; fortunato.bianconi@gmail.com \\ 4 Department of Experimental Medicine, Public Health Section, University of Perugia, 06129 Perugia, Italy; \\ fabrizio.stracci@unipg.it \\ * Correspondence: vienna.ludovini@ospedale.perugia.it; Tel./Fax: +39-075-5783453 \\ + These authors have contributed equally to this work.
}

Received: 17 July 2020; Accepted: 6 August 2020; Published: 12 August 2020

check for updates

\begin{abstract}
Hereditary breast and ovarian cancers are mainly linked to variants in $B R C A 1 / 2$ genes. Recently, data has shown that identification of $B R C A$ variants has an immediate impact not only in cancer prevention but also in targeted therapeutic approaches. This prospective observational study characterized the overall germline $B R C A$ variant and variant of uncertain significance (VUS) frequency and spectrum in individuals affected by breast (BC) or ovarian cancer (OC) and in healthy individuals at risk by sequencing the entire $B R C A$ genes. Of the 363 probands analyzed, $50(13.8 \%)$ were $B R C A 1 / 2$ mutated, $28(7.7 \%)$ at $B R C A 1$ and $23(6.3 \%)$ at $B R C A 2$ gene. The variant c.5266dupC p.(Gln1756Profs) was the most frequent alteration, representing $21.4 \%$ of the $B R C A 1$ variants and $12.0 \%$ of all variants identified. The variant c.6313delA p.(Ile2105Tyrfs) of BRCA2 was the most frequent alteration observed in 6 patients. Interestingly, two new variants were identified in $B R C A 2$. In addition, 25 different VUS were identified; two were reported for the first time in BRCA1 and two in $B R C A 2$. The number of triple-negative $B C s$ was significantly higher in patients with the pathogenic $B R C A 1 / 2$-variant (36.4\%) than in BRCA1/2 VUS (16.0\%) and BRCA1/2 wild-type patients $(10.7 \%)(p<0.001)$. Our study reveals that the overall frequency of $B R C A$ germline variants in the selected high-risk Italian population is about $13.8 \%$. We believe that our results could have significant implications for preventive strategies for unaffected $B R C A$-carriers and effective targeted treatments such as PARP inhibitors for patients with BC or OC.
\end{abstract}

Keywords: $B R C A 1 / 2$ variant carrier; breast cancer; VUS; genetic testing; risk evaluation

\section{Introduction}

Breast cancer (BC) is the most common cancer and leading cause of cancer-related mortality among women worldwide. In Europe, approximately 500,000 women are diagnosed with BC annually, and in 2018, BC cases were responsible for a third of all cancer related deaths (about 130,000) [1]. Most women with breast or ovarian cancer (OC) have a sporadic rather than an inherited cancer. 
However, the majority of hereditary breast and ovarian cancers (HBOC) are due to highly penetrant germline $B R C A$ variants, which are inherited in an autosomal-dominant fashion: breast cancer susceptibility gene 1 (BRCA1) or breast cancer susceptibility gene 2 (BRCA2). In these patients, there are frequently several generations of women affected with $B C$ (often premenopausal) and, in some families, OC as well. The prevalence of $B R C A$ variants varies based on a number of factors, including type of cancer and age at diagnosis. For individuals whose ethnicity is associated with higher variant frequency, particularly Ashkenazi Jews, any personal or family history of $B C$ is sufficient to warrant consideration of $B R C A$ testing. Aside from Ashkenazi Jews, founder variants have also been reported worldwide in populations from the Netherlands, Sweden, Hungary, Iceland, Italy, France, South Africa, Pakistan, Asia, and among French Canadians, Hispanics, and African Americans [2-5].

In a recent study, the incidences of $B C$ and OC were reported to be $72 \%$ or $44 \%$ in $B R C A 1$ carriers and $69 \%$ or $17 \%$ in $B R C A 2$ carriers, respectively [6,7]. Other $B R C A$-associated malignancies such as prostate, male breast and pancreatic cancer may also be observed. Less commonly, BC is due to other hereditary syndromes, such as Li-Fraumeni and Cowden, which are associated with variants in the TP53 and PTEN genes, respectively [8]. BC is the most prevalent cancer type and the first cause of death among women in Italy [9]. International guidelines, in cases of known variants in the family, early-onset or triple-negative cancers and multiple relatives with cancer, suggest referral for genetic counseling [10,11]. In recent years, poly(ADP-ribose) polymerase (PARP) inhibitors have been developed that target $B R C A$ pathogenic variants in various cancer types including breast and ovarian cancers [12]. Thus, the detection of $B R C A$ variants has a relevant impact both in cancer prevention and in targeted treatment. Typically, variant screening has been performed among affected women, selected on the basis of young age at diagnosis or family cancer history. The aim of this study is to determine the overall germline $B R C A$ variant frequency and spectrum in healthy Italian individuals at risk or affected by $B C$ or $O C$ by molecular genetic analysis of regions of $B R C A 1$ and $B R C A 2$ genes.

\section{Materials and Methods}

\subsection{Patients and Samples}

Individuals referring to genetic counseling at the Medical Oncology Division of the S. Maria della Misericordia Hospital (Perugia-Italy) in the years 2010-2016 at risk or with a history of BC or OC were included in the study. This cohort of 363 women/men was selected according to the Italian Medical Oncology Association (AIOM) guidelines [13] based on age at BC/OC onset, number of cancer cases in I- and II degree relatives, and pathological characteristics of BC. Several genetic risk assessment methods are available to estimate the probability of $B R C A$ variant in individuals in order to select them for molecular diagnosis [14]. Genetic testing was performed on all individuals $>18$ years old selected according to the AIOM guidelines and these criteria do not differ from other jurisdictions in Italy.

- Knowledge of pathogenetic mutation in the family

- Males affected by breast cancer

- Women with breast and ovarian cancer

- Women affected by breast cancer $<36$ years old

- Women affected by triple negative breast cancer $<60$ years old

- Women with bilateral breast cancer $<50$ years old

- Women with breast cancer $<50$ years old AND first degree familiarity of:

1. Breast cancer $<50$ years old

2. No-mucinous, no-border line ovarian cancer (all ages)

3. Bilateral breast cancer

4. Male breast cancer 


\section{Pancreatic cancer}

6. Prostate cancer

We chose, however, to utilize BRCAPRO software that is based on Bayes' theorem; this requires data on all first, second and third degree relatives of the family proband and incorporates as prior probabilities incidence rates in the US population, allele variant frequencies and penetrances estimated from studies in families with several BC or OC cases [15-17]. For unaffected individuals we utilized the Cuzick-Tyrer model that, developed for the International Breast Intervention Study (IBIS-1), incorporates the assessment of additional hereditary factors, body mass index, menopausal status and hormone replacement therapy use [18]. We considered it suitable for genetic testing of $B R C A$ variant individuals with an estimated life-time risk of disease $\geq 10 \%$. The study was conducted in accordance with Good Clinical Practice and the ethical principles of the Declaration of Helsinki and approved by the S. Maria della Misericordia Ethics Committee (CE, protocol 2207/2010). We obtained written informed consent from all participants. Clinical data such as age at diagnosis, hystotype, grading, stage, tumor invasiveness, and receptor status were gathered.

St Gallen guidelines were used to classify BC subtype, based on receptor status [19]. Data about a second $\mathrm{BC}$ and/or OC or other malignancies and the family cancer history in I and II degree relatives were also collected.

\subsection{BRCA1/2 Analysis}

Ten milliliters of whole blood mixed with EDTA were collected from each patient. Genomic DNA was extracted from blood using the QIAamp DNA mini kit (Qiagen, Hilden, Germany) and quantified using the Qubit dsDNA BR Assay Kit (ThermoFisher Scientific, M0, Italy). All 23 coding exons of BRCA1 (exons 2 to 24) and 26 coding exons of BRCA2 (exons 2 to 27) were amplified in 33 and 46 amplicons, respectively. The primers were designed to cover all coding exons and adjacent 20 base pair introns. The amplified DNA fragments were sequenced using the BigDyeTerminator v.3.1 cycle sequencing kit (Thermo Fisher Scientific) on a 3500 Genetic analyzer (Applied Biosystems, Foster City, CA, USA). Sequencing chromatograms were analyzed for variant detection using Seqscape software v.2.7 (Applied Biosystems, Foster City, CA, USA). In all cases, samples harboring variants were re-amplified and re-sequenced using the same experimental conditions. All sequences were compared with the BRCA1 (NM_007294.3) and BRCA2 (NM_000059.3) reference sequences for variant detection. To identify gross deletions/insertions not detectable by sequencing on the $B R C A 1 / 2$ genes, we performed the Multiplex Ligation-dependent Probe Amplification (MLPA) using the SALSA P002 BRCA1 and SALSA P045 BRCA2 MLPA probe mix assays (MRC-Holland, Amsterdam, The Netherlands) according to the manufacturer's instructions. Coffalyser V9.4 software (MRC-Holland, Amsterdam, The Netherlands) was used to analyze MLPA results.

\subsection{Variant Classification}

According to the IARC recommendations [20], we classified genetic variants identified into five classes. To annotate $B R C A 1 / 2$ variants we used: databases such as Breast Cancer Information Core (BIC) [21], BRCA Share (formerly Universal Variant Database) [22], Leiden Open Variation Database (LOVD) [23], ClinVar-NCBI Database, and American College of Medical Genetics (ACMG) guidelines [24].

Variants not found in these databases were classified on the basis of their characteristics.

All variants with conflicting interpretation results by ClinVar-NCBI Database were considered as VUSs. The classification of variants initially considered as VUS was subjected to regular updates, by reviewing the literature and publicly available databases to the best of our knowledge, and modified accordingly. Frameshift and nonsense VUS leading to a premature stop codon were considered likely-pathogenic-class 4 and classified in accordance with the ACMG guidelines. All variants were reported according to Human Genome Variation Society nomenclature [25] according to ENIGMA 
(Evidence-based Network for the Interpretation of Germline Mutant Alleles) consortium rules for variant classification to obtain the most recent information on variant reclassifications.

\subsection{Data Collection and Statistical Analysis}

Data were collected using a management system that is integrated with the Umbria Cancer Registry application system [26].

Descriptive statistics of patients' characteristics and sequencing results were presented as median and range for continuous data and as natural frequencies and percentages for categorical data. Pearson Chi-square test or an appropriate Fisher Exact test were used to compare tabular proportions. All data analyses were performed using R software version 3.4.2 (R Foundation for Statistical Computing, Vienna, Austria).

\subsection{Immunohistochemistry Analysis of Breast Tumor Samples}

Tumor immunohistochemical (IHC) analysis was performed for estrogen receptor (ER) (clone 1D5 diluted 1:15), progesterone receptor (PgR) (clone 1A6 diluted 1:15), and Ki-67 (clone MIB1 diluted 1:15) using the automated platform Bond III (Leica Biosystem, MI, Italy). IHC analysis for evaluation of human epidermal growth factor receptor 2 (HER2) status was performed using the HercepTest ${ }^{\mathrm{TM}}$ kit (Dako, Glostrup, Denmark) with an automated system (Autostainer Link 48, Dako) according to the manufacturer's instructions. HER2 status was defined as negative (HercepTest scores of 0 or $1+$ ), doubtful $(2+$ score $)$, and positive $(3 \pm$ score). To confirm HER2 status when IHC results were doubtful, we used Fluorescence in-situ hybridization test using a HER2 FISH PharmDx ${ }^{\mathrm{TM}}$ kit (Dako Glostrup, Denmark), and gene amplification was recorded when the HER2/centromeric probe for chromosome 17 signal ratio was $\geq 2.0$.

\section{Results}

\subsection{Patient Characteristics}

This prospective observational study included 363 Central Italian individuals: 263 (72.4\%) with BC (median age 46 years), 16 (4.4\%) with other tumors, and 84 (23.1\%) with no tumor. Of the 263 BC patients, $217(82.5 \%)$ had a first BC, $44(16.7 \%)$ a second BC and $2(0.8 \%)$ had subsequent three BC. Among the 10 patients with OC, 3 had initial OC and 7 had a second OC after BC. The BRCA2 pathogenic variants were significantly prevalent in patients with initial $\mathrm{BC}(p=0.006$, Fisher Exact test) while $B R C A 1$ pathogenic variants were significantly present in patients with $\mathrm{OC}(p<0.001$, Fisher Exact test). BC and OC patient tumor characteristics are summarized in Table 1. The majority of individuals genotyped with no a priori data on familial variant 269/363 (74.1\%) were tested because of personal history of cancer while 94/363 (25.9\%) were referred for oncogenetic counselling and genotyping because of a family history suggestive of inherited predisposition to cancer. 
Table 1. Population Characteristics.

\begin{tabular}{|c|c|c|c|c|c|c|c|c|c|c|c|}
\hline & & & \multicolumn{3}{|c|}{ BRCA1 } & \multicolumn{3}{|c|}{ BRCA2 } & \multicolumn{3}{|c|}{ BRCA1/2 } \\
\hline & & & $\begin{array}{l}\text { Pathogenic/Likely } \\
\text { Pathogenic Variants }\end{array}$ & vus & $\begin{array}{l}\text { No Pathogenic } \\
\text { Variants }\end{array}$ & $\begin{array}{l}\text { Pathogenic/Likely } \\
\text { Pathogenic Variants }\end{array}$ & vus & $\begin{array}{l}\text { No Pathogenic } \\
\text { Variants }\end{array}$ & $\begin{array}{l}\text { Pathogenic/Likely } \\
\text { Pathogenic Variants }\end{array}$ & vus & $\begin{array}{l}\text { No Pathogenic } \\
\text { Variants }\end{array}$ \\
\hline $\begin{array}{l}* * * \text { Overall Central Italian } \\
\text { individuals (N. \%) }\end{array}$ & & $\begin{array}{c}363 \\
(100.0)\end{array}$ & $\begin{array}{l}28 \\
(7.7)\end{array}$ & $\begin{array}{c}9 \\
(2.5)\end{array}$ & $\begin{array}{c}326 \\
(89.8)\end{array}$ & $\begin{array}{l}23 \\
(6.3)\end{array}$ & $\begin{array}{c}21 \\
(5.8)\end{array}$ & $\begin{array}{c}319 \\
(87.9)\end{array}$ & $\begin{array}{r}50 * * \\
(13.8)\end{array}$ & $\begin{array}{l}28 \\
(7.7)\end{array}$ & $\begin{array}{l}285 \\
(78.5)\end{array}$ \\
\hline \multirow[t]{3}{*}{ Age at diagnosis, years } & $\begin{array}{c}\text { Median } \\
\text { Range (Min-Max) }\end{array}$ & $\begin{array}{c}47 \\
(19-84)\end{array}$ & $\begin{array}{c}49 \\
(22-69)\end{array}$ & $\begin{array}{c}54 \\
(37-74)\end{array}$ & $\begin{array}{c}47 \\
(19-84)\end{array}$ & $\begin{array}{c}48 \\
(19-84)\end{array}$ & $\begin{array}{c}50 \\
(27-72)\end{array}$ & $\begin{array}{c}47 \\
(19-81)\end{array}$ & $\begin{array}{c}48 \\
(19-84)\end{array}$ & $\begin{array}{c}51 \\
(27-74)\end{array}$ & $\begin{array}{c}47 \\
(19-81)\end{array}$ \\
\hline & $p$-value * & & & 0.165 & & & 0.444 & & & 0.09 & \\
\hline & & $\begin{array}{l}\mathrm{N} \\
(\%)\end{array}$ & & & & & & & & & \\
\hline \multicolumn{12}{|l|}{ Sex } \\
\hline & Female & $\begin{array}{r}351 \\
(97.7)\end{array}$ & $\begin{array}{c}27 \\
(96.4)\end{array}$ & $\begin{array}{c}8 \\
(88.9) \\
\end{array}$ & $\begin{array}{l}316 \\
(96.9)\end{array}$ & $\begin{array}{c}22 \\
(95.7)\end{array}$ & $\begin{array}{c}21 \\
(100.0)\end{array}$ & $\begin{array}{c}308 \\
(96.6)\end{array}$ & $\begin{array}{c}48 \\
(96.0)\end{array}$ & $\begin{array}{c}27 \\
(96.4)\end{array}$ & $\begin{array}{c}276 \\
(96.8)\end{array}$ \\
\hline & Male & $\begin{array}{l}12 \\
(3.3)\end{array}$ & $\begin{array}{c}1 \\
(3.6)\end{array}$ & $\begin{array}{c}1 \\
(11.1)\end{array}$ & $\begin{array}{l}10 \\
(3.1)\end{array}$ & $\begin{array}{c}1 \\
(4.3)\end{array}$ & $\begin{array}{c}0 \\
(0.0)\end{array}$ & $\begin{array}{l}11 \\
(3.4)\end{array}$ & $\begin{array}{c}2 \\
(4.0)\end{array}$ & $\begin{array}{c}1 \\
(3.6)\end{array}$ & $\begin{array}{c}9 \\
(3.2)\end{array}$ \\
\hline & $p$-value * & & & 0.411 & & & 0.665 & & & 0.961 & \\
\hline \multicolumn{12}{|l|}{ Tumor Type } \\
\hline \multicolumn{12}{|l|}{ BC } \\
\hline & First BC & $\begin{array}{c}217 \\
(59.9) \\
\end{array}$ & $\begin{array}{c}15 \\
(53.6) \\
\end{array}$ & $\begin{array}{c}5 \\
(55.6) \\
\end{array}$ & $\begin{array}{l}197 \\
(60.4) \\
\end{array}$ & $\begin{array}{c}11 \\
(47.8)\end{array}$ & $\begin{array}{c}13 \\
(61.9) \\
\end{array}$ & $\begin{array}{l}193 \\
(60.5) \\
\end{array}$ & $\begin{array}{l}25 \\
(50.0) \\
\end{array}$ & $\begin{array}{c}14 \\
(57.1)\end{array}$ & $\begin{array}{c}176 \\
(61.8) \\
\end{array}$ \\
\hline & Second BC & $\begin{array}{c}44 \\
(12.1)\end{array}$ & $\begin{array}{c}4 \\
(14.3) \\
\end{array}$ & $\begin{array}{c}2 \\
(22.2)\end{array}$ & $\begin{array}{c}38 \\
(11.7)\end{array}$ & $\begin{array}{c}3 \\
(13.0)\end{array}$ & $\begin{array}{c}7 \\
(33.3)\end{array}$ & $\begin{array}{c}34 \\
(10.7)\end{array}$ & $\begin{array}{c}7 \\
(14.0)\end{array}$ & $\begin{array}{c}9 \\
(32.2)\end{array}$ & $\begin{array}{c}28 \\
(9.8)\end{array}$ \\
\hline & Third BC & $\begin{array}{c}2 \\
(0.5) \\
\end{array}$ & $\begin{array}{c}0 \\
(0.0) \\
\end{array}$ & $\begin{array}{c}0 \\
(0.0) \\
\end{array}$ & $\begin{array}{c}2 \\
(0.6) \\
\end{array}$ & $\begin{array}{c}1 \\
(4.4) \\
\end{array}$ & $\begin{array}{c}0 \\
(0.0) \\
\end{array}$ & $\begin{array}{c}1 \\
(0.3) \\
\end{array}$ & $\begin{array}{c}1 \\
(2.0)\end{array}$ & $\begin{array}{c}0 \\
(0.0) \\
\end{array}$ & $\begin{array}{c}1 \\
(0.4) \\
\end{array}$ \\
\hline & Other tumors & $\begin{array}{c}16 \\
(4.4)\end{array}$ & $\begin{array}{c}2 \\
(7.1)\end{array}$ & $\begin{array}{c}1 \\
(11.1)\end{array}$ & $\begin{array}{c}13 \\
(4.0) \\
\end{array}$ & $\begin{array}{c}2 \\
(8.7) \\
\end{array}$ & $\begin{array}{c}1 \\
(4.8)\end{array}$ & $\begin{array}{l}13 \\
(4) \\
\end{array}$ & $\begin{array}{c}4 \\
(8.0) \\
\end{array}$ & $\begin{array}{c}2 \\
(7.1) \\
\end{array}$ & $\begin{array}{c}10 \\
(3.5)\end{array}$ \\
\hline & No tumors & $\begin{array}{c}84 \\
(23.1)\end{array}$ & $\begin{array}{c}7 \\
(25)\end{array}$ & $\begin{array}{c}1 \\
(11.1)\end{array}$ & $\begin{array}{c}76 \\
(23.3)\end{array}$ & $\begin{array}{c}6 \\
(26.1)\end{array}$ & $\begin{array}{c}0 \\
0.0)\end{array}$ & $\begin{array}{c}78 \\
(24.5)\end{array}$ & $\begin{array}{c}13 \\
(26.0)\end{array}$ & $\begin{array}{c}1 \\
(3.6)\end{array}$ & $\begin{array}{c}70 \\
(24.5)\end{array}$ \\
\hline & $p$-value * & & & 0.898 & & & 0.006 & & & 0.006 & \\
\hline \multicolumn{12}{|l|}{ OC } \\
\hline & First OC & $\begin{array}{c}3 \\
(0.8)\end{array}$ & $\begin{array}{c}2 \\
(7.1)\end{array}$ & $\begin{array}{c}0 \\
(0.0)\end{array}$ & $\begin{array}{c}1 \\
(0.3)\end{array}$ & $\begin{array}{c}0 \\
(0.0)\end{array}$ & $\begin{array}{c}0 \\
(0.0)\end{array}$ & $\begin{array}{c}3 \\
(0.9)\end{array}$ & $\begin{array}{c}2 \\
(4.0)\end{array}$ & $\begin{array}{c}0 \\
(0.0)\end{array}$ & $\begin{array}{c}1 \\
(0.3)\end{array}$ \\
\hline & Both $\mathrm{BC}$ and $\mathrm{OC}$ & $\begin{array}{c}7 \\
(1.9) \\
\end{array}$ & $\begin{array}{c}5 \\
(17.9) \\
\end{array}$ & $\begin{array}{c}0 \\
(0.0) \\
\end{array}$ & $\begin{array}{c}2 \\
(0.6) \\
\end{array}$ & $\begin{array}{c}0 \\
(0.0) \\
\end{array}$ & $\begin{array}{c}1 \\
(4.8) \\
\end{array}$ & $\begin{array}{c}6 \\
(1.9) \\
\end{array}$ & $\begin{array}{c}5 \\
(10.0) \\
\end{array}$ & $\begin{array}{c}1 \\
(3.6) \\
\end{array}$ & $\begin{array}{c}1 \\
(0.3) \\
\end{array}$ \\
\hline & No & $\begin{array}{c}269 \\
(96.4)\end{array}$ & $\begin{array}{c}21 \\
(75.0)\end{array}$ & $\begin{array}{c}9 \\
(100.0)\end{array}$ & $\begin{array}{c}323 \\
(99.1)\end{array}$ & $\begin{array}{c}23 \\
(100.0)\end{array}$ & $\begin{array}{c}20 \\
(95.2)\end{array}$ & $\begin{array}{c}310 \\
(97.2)\end{array}$ & $\begin{array}{c}43 \\
(86.0)\end{array}$ & $\begin{array}{c}27 \\
(96.4)\end{array}$ & $\begin{array}{c}283 \\
(99.3)\end{array}$ \\
\hline & $p$-value * & & & $<0.001$ & & & 0.779 & & & $<0.001$ & \\
\hline
\end{tabular}

Abbreviations: BC, breast cancer; OC, ovarian cancer; VUS, variant of uncertain significance. * Pearson Chi-square test or the Fisher Exact test, as appropriate. ${ }^{* *}$ One patients possess the

pathogenic variants of the both $B R C A 1$ and $B R C A 2$ genes simultaneously (ID 606). ${ }^{* * *}$ the individuals were all Caucasians. 


\subsection{BRCA Variants and Patient Characteristics}

A total of 363 oncogenetic genotyping results were performed in the present study, 351 in females (97.7\%) and $12(3.3 \%)$ in males. Overall, 50/363 (13.8\%) genotyping individuals carried one pathogenic/likely pathogenic variant in either $B R C A$ gene, including $28(7.7 \%)$ pathogenic/likely pathogenic $B R C A 1$ variants and $23(6.3 \%)$ pathogenic/likely pathogenic $B R C A 2$ variants (Table 2A). One patient had two variants in both BRCA1 and BRCA2 genes (sample ID 606, Table 2A). Thirteen of $50(26.0 \%)$ variants found were carried in people with no history of cancer and $38 / 50$ variants $(76.0 \%)$ were detected in patients affected by BC. Of the BC BRCA-mutated patients, $21(56.7 \%)$ were affected by a variant of $B R C A 1$ and $17(45.3 \%)$ by a $B R C A 2$ variant. Of 13 women or men without personal history of cancer, $7(53.8 \%)$ were affected by variants of $B R C A 1$ and $6(46.2 \%)$ by variants of $B R C A 2$. On the whole, the majority of $B R C A$ pathogenic variants were reported to be in exon 11 for both genes: $10(43.5 \%)$ variants in exon 11 of $B R C A 1$ and 13 (56.5\%) of $B R C A 2$ gene, respectively. All detected pathogenic/likely pathogenic variants with the exception of three in splice sites of $B R C A 2$ gene and three variants missense of $B R C A 1$ gene, the cause being either termination or a frameshift in $B R C A$ proteins. Five $B R C A$-variant carriers $(17.9 \%)$ were affected from both $B C$ and OC. Of seven patients presented with bilateral $B C(14.6 \%)$, three $B R C A 1$ and four $B R C A 2$ pathogenic variants were found.

\subsection{Cohort Spectrum and Variant Detection Rate}

Table 2A lists the pathogenic/likely-pathogenic variants detected in the BRCA1 and BRCA2 genes, and Table $2 \mathrm{~B}$ shows the $B R C A 1$ and $B R C A 2$ VUS variants as well as their frequencies. We found 14 different pathogenic/likely-pathogenic variants in BRCA1 gene and 16 in BRCA2 gene. Overall, of the 30 pathogenic/likely-pathogenic variants, 2 (6.6\%) were novel variants in exon 17 of $B R C A 2$ (c.7828_7834delGTGGATC p.(Val2610fs); c.7852_7862delATTTGGGTTTA, p.(Ile2618fs)) not previously reported in BIC, LOVD, ClinVar-NCBI Database, BRCA-Share or any published literature. Besides the detrimental variant detected, 9 and 16 VUS were identified in the $B R C A 1$ and $B R C A 2$ genes, respectively. Of these 25 BRCA1/2 VUS, 2 are reported here for the first time in BRCA1 (c.4986 + 47A > G (IVS16 + $47 \mathrm{~A}>\mathrm{G}$ ) in exon 16; c.5407-72delAAAA (IVS22-72delAAAA) in exon 23) and 2 in BRCA2 (c.4504C > A p.(Gln1502Lys) in exon 11; c.7618-11delATTTT (IVS15-11delATTTT) in exon 16). The most frequent VUS variant detected in exon 11 of $B R C A 2$ c.5972C $>$ T p.(Ala1991Val) was observed in five patients. Seven women presented at the same time a VUS and a pathogenic variant, three patients with VUS resulted affected by both $\mathrm{OC}$ and $\mathrm{BC}$ and six patients had bilateral $\mathrm{BC}$.

\subsection{Recurrent Pathogenic/Likely-Pathogenic BRCA1/2 Variants}

Of the 30 distinct pathogenic/likely-pathogenic $B R C A$ variants in our patient cohort, 23 were observed only once; 5 in $B R C A 1$ and 2 in $B R C A 2$ variants were detected in at least two or more. These seven variants were detected in $23.3 \%$ of all patients with pathogenic $B R C A$ variant. The most frequent pathogenic variant detected in BRCA1 c.5266dupC p.(Gln1756Profs) exon 20 and BRCA2 c.6313delA p.(Ile2105Tyrfs) exon 11, was observed in six patients, respectively (Table 2A). 
Table 2. (A) List of $B R C A 1$ and $B R C A 2$ pathogenic/likely pathogenic variants detected in 50 Central Italian individuals. (B) List of $B R C A 1$ and $B R C A 2$ Variants of Uncertain Significance (VUS) variants detected in 33 Central Italian individuals *.

\begin{tabular}{|c|c|c|c|c|c|c|c|c|c|}
\hline Sample ID & Gene & Exon/Intron & $\begin{array}{c}\text { HGVS cDNA } \\
\text { (BRCA1 NM_007294.3) } \\
\text { (BRCA2 NM_000059.3) }\end{array}$ & HGVS Protein & Variant Type & $\begin{array}{c}\text { IARC } \\
\text { Classification }\end{array}$ & ClinVar & $\begin{array}{l}\text { BRCA Share- } \\
\text { BIC-LOVD }\end{array}$ & $\mathbf{N}$. \\
\hline 66,101 & $B R C A 1$ & 2 & c.68_69delAG & p.(Glu23Valfs) & Frameshift deletion & Class-5 & Pathogenic & Pathogenic & 2 \\
\hline 315 & $B R C A 1$ & 3 & c. $116 \mathrm{G}>\mathrm{A}$ & p.(Cys39Tyr) & Missense & Class- 5 & Pathogenic & Pathogenic & 1 \\
\hline 909 & $B R C A 1$ & 5 & c. $181 \mathrm{~T}>\mathrm{G}$ & p.(Cys61Gly) & Missense & Class-5 & Pathogenic & Pathogenic & 1 \\
\hline 403 & $B R C A 1$ & 11 & c. $1999 \mathrm{C}>\mathrm{T}$ & p.(Gln667Ter) & Nonsense & Class- 5 & Pathogenic & Pathogenic & 1 \\
\hline 833 & $B R C A 1$ & 11 & c.3228_3229delAG & p.(Gly1077Alafs) & Frameshift deletion & Class- 5 & Pathogenic & Pathogenic & 1 \\
\hline $265,287,471,524$ & $B R C A 1$ & 11 & c.2406_2409delGAGT & p.(Gln804Valfs) & Frameshift deletion & Class-5 & Pathogenic & Pathogenic & 4 \\
\hline $475,606,1341$ & $B R C A 1$ & 11 & c.3326-3329delAAAA & p.(Lys1109Serfs) & Frameshift deletion & Class-5 & Pathogenic & Pathogenic & 3 \\
\hline 223 & $B R C A 1$ & 11 & c.3599_3600delAG & p.(Gln1200Argfs) & Frameshift deletion & Class- 5 & Pathogenic & Pathogenic & 1 \\
\hline 443 & $B R C A 1$ & 12 & c. $4117 \mathrm{G}>\mathrm{T}$ & p.(Glu1373Ter) & Nonsense & Class- 5 & Pathogenic & Pathogenic & 1 \\
\hline 161 & $B R C A 1$ & 16 & c.4964_4982del19 & p.(Ser1655Tyrfs) & Frameshift deletion & Class-5 & Pathogenic & Pathogenic & 1 \\
\hline $270,300,358,1011$ & BRCA1 & 17 & c.5062_5064delGTT & p.(Val1688del) & Inframe deletion & Class- 5 & Pathogenic & Pathogenic & 4 \\
\hline 50 & $B R C A 1$ & 18 & c. $5096 \mathrm{G}>\mathrm{A}$ & p.(Arg1699Gln) & Missense & Class-5 & Pathogenic & Pathogenic & 1 \\
\hline $47,150,746,938,943,609$ & BRCA1 & 20 & c.5266dupC & p.(Gln1756Profs) & Frameshift insertion & Class- 5 & Pathogenic & Pathogenic & 6 \\
\hline 932 & $B R C A 1$ & 23 & c. $5445 \mathrm{G}>\mathrm{A}$ & p.(Trp1815Ter) & Nonsense & Class-5 & Pathogenic & Pathogenic & 1 \\
\hline 616 & $B R C A 2$ & 2 & c. $67+1 \mathrm{G}>\mathrm{A}$ & - & Splicing & Class- 5 & Pathogenic & Pathogenic & 1 \\
\hline 606 & $B R C A 2$ & 8 & c. $632-2 A>G$ & - & Splicing & Class- 5 & Pathogenic & Pathogenic & 1 \\
\hline 289 & $B R C A 2$ & 8 & c.658_659delGT & p.(Val220Ilefs) & Frameshift deletion & Class-5 & Pathogenic & Pathogenic & 1 \\
\hline 426 & $B R C A 2$ & 11 & c.3919delG & p.(Glu1307Lysfs) & Frameshift deletion & Class- 5 & Pathogenic & Pathogenic & 1 \\
\hline 352 & $B R C A 2$ & 11 & c.4284dupT & p.(Gln1429Serfs) & Frameshift deletion & Class-5 & Pathogenic & Pathogenic & 1 \\
\hline 959 & $B R C A 2$ & 11 & c. $5645 \mathrm{C}>\mathrm{A}$ & p.(Ser1882Ter) & Nonsense & Class-5 & Pathogenic & Pathogenic & 1 \\
\hline $865,946,1004$ & $B R C A 2$ & 11 & c.5722_5723delCT & p.(Leu1908Argfs) & Frameshift deletion & Class -5 & Pathogenic & Pathogenic & 3 \\
\hline 424 & $B R C A 2$ & 11 & c.6039delA & p.(Val2014Tyrfs) & Frameshift deletion & Class-5 & Pathogenic & Pathogenic & 1 \\
\hline $48,78,291,564,614,615$ & $B R C A 2$ & 11 & c.6313delA & p.(Ile2105Tyrfs) & Frameshift deletion & Class- -5 & Pathogenic & Pathogenic & 6 \\
\hline
\end{tabular}


Table 2. Cont

\begin{tabular}{|c|c|c|c|c|c|c|c|c|c|}
\hline Sample ID & Gene & Exon/Intron & $\begin{array}{c}\text { HGVS cDNA } \\
\text { (BRCA1 NM_007294.3) } \\
\text { (BRCA2 NM_000059.3) }\end{array}$ & HGVS Protein & Variant Type & $\begin{array}{c}\text { IARC } \\
\text { Classification }\end{array}$ & ClinVar & $\begin{array}{l}\text { BRCA Share- } \\
\text { BIC-LOVD }\end{array}$ & $\mathbf{N}$ \\
\hline 618 & $B R C A 2$ & 17 & c.7828_7834delGTGGATC & p.(Val2610fs) & Frameshift deletion & Class- 4 & - & - & 1 \\
\hline 367 & $B R C A 2$ & 17 & c.7852_7862delATTTGGGTTTA & p.(Ile2618fs) & Frameshift deletion & Class-4 & - & - & 1 \\
\hline 260 & $B R C A 2$ & 18 & c. $8174 \mathrm{G}>\mathrm{A}$ & p.(Trp2725Ter) & Nonsense & Class-5 & Pathogenic & Pathogenic & 1 \\
\hline 393 & $B R C A 2$ & 19 & c. $8487+1 \mathrm{G}>\mathrm{A}$ & - & Splicing & Class-5 & Pathogenic & UV/Pathogenic & 1 \\
\hline 295 & $B R C A 2$ & 20 & c.8537_8538delAG & p.(Glu2846Glyfs) & Frameshift deletion & Class- 5 & Pathogenic & Pathogenic & 1 \\
\hline 640 & $B R C A 2$ & 22 & c. $8878 \mathrm{C}>\mathrm{T}$ & p.(Gln2960Ter) & Nonsense & Class-5 & Pathogenic & Pathogenic & 1 \\
\hline 571 & $B R C A 2$ & 22 & c.8930delA & p.(Tyr2977Phefs) & Frameshift deletion & Class-5 & Pathogenic & Pathogenic & 1 \\
\hline \multicolumn{10}{|c|}{ Table 2 (B) List of $B R C A 1$ and $B R C A 2$ Variant of Uncertain Significance (VUS) Variants Detected in 33 Central Italian Individuals } \\
\hline Sample ID & Gene & Exon/Intron & $\begin{array}{c}\text { HGVS cDNA } \\
\text { (BRCA1 NM_007294.3) } \\
\text { (BRCA2 NM_000059.3) }\end{array}$ & HGVS Protein & Variant Type & $\begin{array}{c}\text { IARC } \\
\text { Classification }\end{array}$ & Clin Var & $\begin{array}{c}\text { BRCA } \\
\text { Share-BIC-LOVD }\end{array}$ & $\mathbf{N}$ \\
\hline 879 & BRCA1 & 2 & $\begin{array}{c}\text { c.-77delTGT } \\
\text { (IVS0-77delTGT) }\end{array}$ & - & Intron & Class-3 & - & - & 1 \\
\hline 733 & $B R C A 1$ & 7 & c. $335 \mathrm{~A}>\mathrm{G}$ & p.(Asn112Ser) & missense & Class-3 & - & VUS & 1 \\
\hline 632 & $B R C A 1$ & 11 & c. $734 \mathrm{~A}>\mathrm{T}$ & p.(Asp245Val) & missense & Class-3 & VUS & VUS & 1 \\
\hline 635 & $B R C A 1$ & 11 & c. $3711 \mathrm{~A}>\mathrm{G}$ & p.(Ile1237Met) & missense & Class-3 & VUS & VUS & 1 \\
\hline 303 & $B R C A 1$ & 12 & c. $4132 \mathrm{G}>\mathrm{A}$ & p.(Val1378Ile) & missense & Class-3 & VUS & VUS & 1 \\
\hline 1013 & $B R C A 1$ & 16 & $\begin{array}{l}\text { c. } 4986+47 \mathrm{~A}>\mathrm{G} \\
\text { (IVS16+47A > G) }\end{array}$ & - & Intron & Class-3 & - & - & 1 \\
\hline 635 & $B R C A 1$ & 16 & c. $4843 \mathrm{G}>\mathrm{A}$ & p.(Ala1615Thr) & missense & Class-3 & VUS & VUS & 1 \\
\hline 272,478 & $B R C A 1$ & 20 & $\begin{array}{c}\text { c.5277 + 60_5277 + } \\
\text { 61insGTATTCCACTCC }\end{array}$ & - & Intron & Class-3 & VUS & Benign/VUS & 2 \\
\hline 1012 & $B R C A 1$ & 23 & c.5407-72delAAAA & - & Intron & Class-3 & - & - & 1 \\
\hline 527 & $B R C A 2$ & 2 & $\begin{array}{l}\text { c. } 67+62 \mathrm{~T}>\mathrm{G} \\
\text { (IVS2+62T>G) }\end{array}$ & - & Intron & Class-3 & VUS & Benign/VUS & 1 \\
\hline 553 & $B R C A 2$ & 10 & c. $1181 \mathrm{~A}>\mathrm{C}$ & p.(Glu394Ala) & missense & Class-3 & VUS & VUS & 1 \\
\hline 886,930 & $B R C A 2$ & 11 & c. $4928 \mathrm{~T}>\mathrm{C}$ & p.(Val1643Ala) & missense & Class-3 & VUS & VUS & 2 \\
\hline 633 & $B R C A 2$ & 11 & c. $4504 \mathrm{C}>\mathrm{A}$ & p.(Gln1502Lys) & missense & Class-3 & - & - & 1 \\
\hline
\end{tabular}


Table 2. Cont.

\begin{tabular}{|c|c|c|c|c|c|c|c|c|c|}
\hline Sample ID & Gene & Exon/Intron & $\begin{array}{c}\text { HGVS cDNA } \\
\text { (BRCA1 NM_007294.3) } \\
\text { (BRCA2 NM_000059.3) }\end{array}$ & HGVS Protein & Variant Type & $\begin{array}{c}\text { IARC } \\
\text { Classification }\end{array}$ & Clin Var & $\begin{array}{c}\text { BRCA } \\
\text { Share-BIC-LOVD }\end{array}$ & $\mathbf{N}$ \\
\hline $399,532,558,635,679$ & $B R C A 2$ & 11 & c. $5972 \mathrm{C}>\mathrm{T}$ & p.(Ala1991Val) & missense & Class-3 & VUS & VUS & 5 \\
\hline 212,309 & $B R C A 2$ & 11 & c. $6131 \mathrm{G}>\mathrm{C}$ & p.(Gly2044Ala) & missense & Class-3 & VUS & VUS & 2 \\
\hline 423 & $B R C A 2$ & 11 & c. $6441 \mathrm{C}>\mathrm{G}$ & p.His2147Gln) & missense & Class-3 & VUS & VUS & 1 \\
\hline 259,296 & $B R C A 2$ & 11 & c. $6461 \mathrm{~A}>\mathrm{C}$ & p.(Tyr2154Ser) & missense & Class-3 & VUS & VUS & 2 \\
\hline 752 & $B R C A 2$ & 11 & c. $6641 \mathrm{C}>\mathrm{T}$ & p.(Thr2214Ile) & missense & Class-3 & VUS & VUS & 1 \\
\hline 518 & $B R C A 2$ & 15 & c. $7505 \mathrm{G}>\mathrm{A}$ & p.(Arg2502His) & missense & Class-3 & VUS & VUS & 1 \\
\hline 367 & $B R C A 2$ & 16 & c.7618-11delATTTT & - & Intron & Class-3 & - & - & 1 \\
\hline 571 & $B R C A 2$ & 25 & c. $9275 \mathrm{~A}>\mathrm{G}$ & p.(Tyr3092Cys) & missense & Class-3 & VUS & VUS & 1 \\
\hline 1012 & $B R C A 2$ & 25 & c. $9501+3 \mathrm{~A}>\mathrm{T}$ & - & Intron & Class-3 & VUS & VUS & 1 \\
\hline 786 & $B R C A 2$ & 26 & c. $9648+84 \mathrm{G}>\mathrm{A}$ & - & Intron & Class-3 & VUS & $\begin{array}{c}\text { Likely } \\
\text { Benign/VUS }\end{array}$ & 1 \\
\hline 64 & $B R C A 2$ & 27 & c. $10024 \mathrm{G}>\mathrm{A}$ & p.(Glu3342Lys) & missense & Class-3 & VUS & VUS & 1 \\
\hline 1016 & $B R C A 2$ & 27 & c.10095delinsGAATTATATCT & p.(Ser3366fs) & Frameshift deletion & Class-3 & VUS & Benign/VUS & 1 \\
\hline
\end{tabular}

Abbreviations: HGVS, Human Genome Variation Society; cDNA, coding DNA. IARC, International Agency for Research on Cancer; BIC, Breast Cancer Variant Data Base; LOVD, Leiden

Open Variation Database; VUS, Variant of Uncertain Significance. ${ }^{* * *}$ the individuals were all Caucasians. 


\subsection{Characteristics of Breast Cancer in BRCA Carrier Patients}

Table 3 describes the characteristics of $\mathrm{BC}$ in patients with pathogenic/likely pathogenic BRCA1/2 variants in comparison with patients with $B R C A 1 / 2-V U S$ and without $B R C A 1 / 2$ variants. Median age of the 33 patients with pathogenic/likely pathogenic BRCA1/2 variant was 46 years (range 27-65). The most frequent histology was ductal $(n=21,63.6 \%)$, followed by lobular in seven $(21.2 \%)$ patients and other invasive histotypes in five (15.2\%) $(p=0.005$, Fisher Exact test). VUS BRCA2 variants were observed with significant differences in patients with invasive tumor with respect to patients with in situ carcinoma (70\% vs. 30\% respectively, $p=0,014$ Fisher Exact test). According to surrogate definitions of intrinsic subtypes of breast cancer, $36.4 \%$ of tumors were classified as triple negative, $45.5 \%$ as luminal a-like breast cancer and $3.0 \%$ as luminal b-like. The number of triple-negative BCs (TNBCs) was significantly higher in patients with pathogenic BRCA1/2-variant (36.4\%) than in BRCA1/2 VUS $(16.0 \%)$ and $B R C A 1 / 2$ wild type patients $(10.7 \%)(p<0.001$, Fisher Exact test). No enriched HER-2 was found in patients with pathogenic BRCA1/2 variant. In situ carcinoma was significantly observed in $32 \%$ of patients with $B R C A 1 / 2$ VUS with respect to the $11.2 \%$ of patients without $B R C A 1 / 2$ variant ( $p=0.005$, Fisher Exact test). The pathogenic $B R C A 1 / 2$ variant was observed more often in patients with high Ki67 (81.8\%) than in those with BRCA1/2-VUS (44.0\%) and in those without BRCA1/2 variant $(52.7 \%)(p=0.008$, Fisher Exact test). No significant differences were detected in terms of median age, stage, grading, and exitus. An example is shown in Figure 1: the family members of the proband harboring the pathogenic variant c.6313delA in the BRCA2 gene. As shown in the pedigree, the proband diagnosed with bilateral breast cancer at the age of 38 carried the pathogenic variant in BRCA2. She had a first-degree relative with both ovarian and breast cancer and a second-degree relative with bilateral breast cancer. Estimated variant probability for $B R C A 1 / 2$ before genetic testing was $26.6 \%$ by Myriad and $18.4 \%$ by BRCAPRO. Genetic testing was performed on her two cousins with breast cancer who carried a $B R C A 2$ gene with the same pathogenic variant. Her two daughters without breast cancer had the same pathogenic variant.

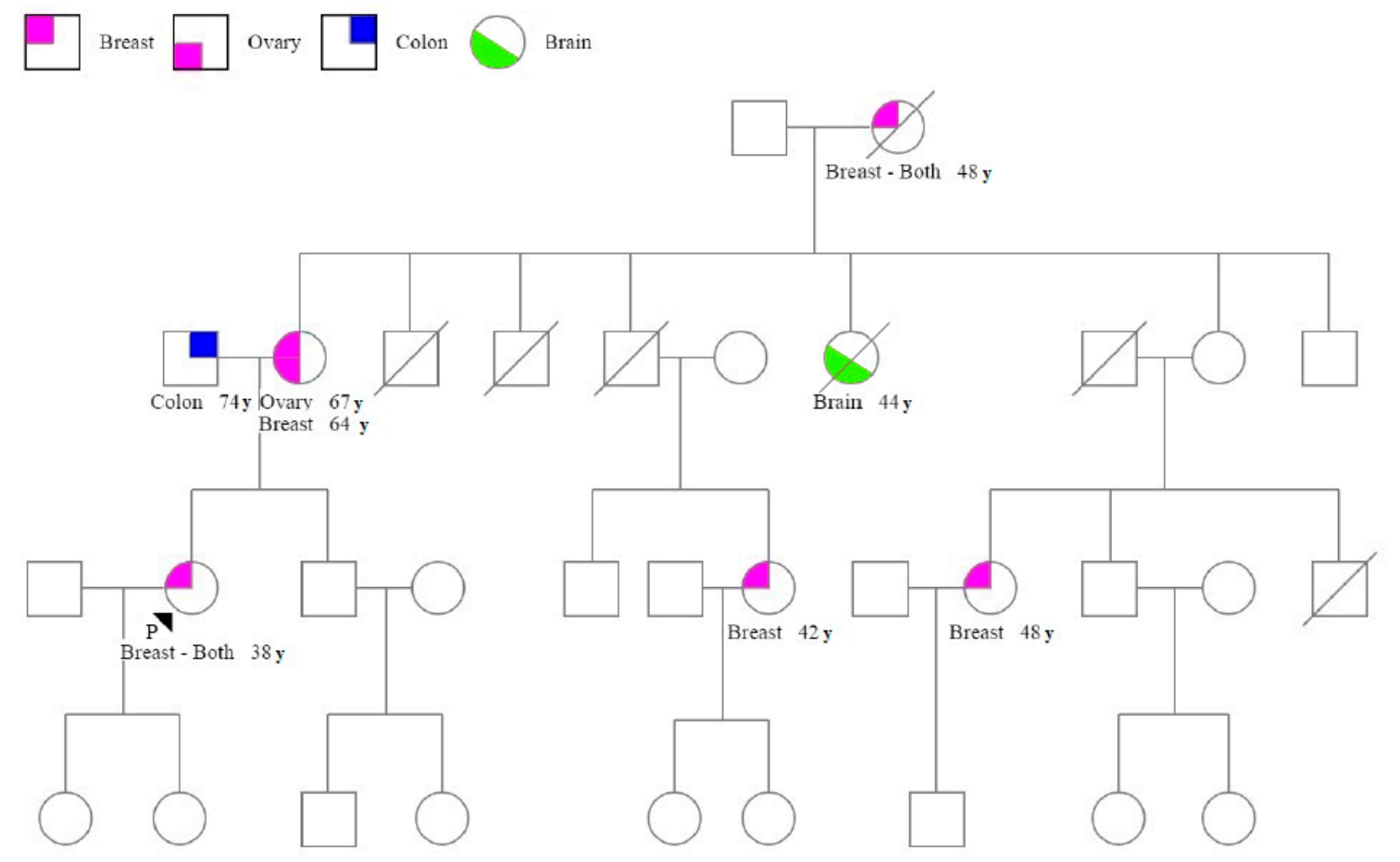

Figure 1. Pedigree of patient ID 48 with c.6313delA p.(Ile2105Tyrfs) pathogenic variant in the BRCA2 gene. The proband is indicated by a black arrow. Cancer Type and age at cancer diagnosis is indicated in the legend. Symbols: squares = males, circles = females; quadrant shading = cancer affected; slash through square or circle $=$ deceased. 
Table 3. Clinical features and $B R C A$ status in $\mathrm{BC}$

\begin{tabular}{|c|c|c|c|c|c|c|c|c|c|c|c|}
\hline & & & \multicolumn{3}{|c|}{ BRCA1 } & \multicolumn{3}{|c|}{ BRCA2 } & \multicolumn{3}{|c|}{ BRCA1/2 } \\
\hline & & & Variants & VUS & $\begin{array}{l}\text { No Pathogenic } \\
\text { Variants }\end{array}$ & Variants & VUS & $\begin{array}{l}\text { No Pathogenic } \\
\text { Variants }\end{array}$ & Variants & VUS & $\begin{array}{l}\text { No Pathogenic } \\
\text { Variants }\end{array}$ \\
\hline $\begin{array}{c}{ }^{* * *} \text { Overall Central } \\
\text { Italian individuals } \\
\text { (N. \%) }\end{array}$ & & $\begin{array}{c}263 \\
(100)\end{array}$ & $\begin{array}{c}19 \\
(7.2)\end{array}$ & $\begin{array}{c}7 \\
(2.7)\end{array}$ & $\begin{array}{c}237 \\
(90.1)\end{array}$ & $\begin{array}{l}15 \\
(5.7)\end{array}$ & $\begin{array}{c}20 \\
(7.6)\end{array}$ & $\begin{array}{c}228 \\
(86.7)\end{array}$ & $\begin{array}{l}33 \text { ** } \\
(12.6)\end{array}$ & $\begin{array}{c}25 \\
(9.5)\end{array}$ & $\begin{array}{c}205 \\
(77.9)\end{array}$ \\
\hline \multirow[t]{7}{*}{ Age at diagnosis, years } & $\begin{array}{c}\text { Median } \\
\text { Range(Min-Max) }\end{array}$ & $\begin{array}{c}46 \\
(27-77)\end{array}$ & $\begin{array}{c}47 \\
(31-63)\end{array}$ & $\begin{array}{c}7 \\
(37-58)\end{array}$ & $\begin{array}{c}46 \\
(27-77)\end{array}$ & $\begin{array}{c}44 \\
(27-65)\end{array}$ & $\begin{array}{c}50 \\
(34-68)\end{array}$ & $\begin{array}{c}46 \\
(27-77)\end{array}$ & $\begin{array}{c}46 \\
(27-65)\end{array}$ & $\begin{array}{c}48 \\
(34-68)\end{array}$ & $\begin{array}{c}46 \\
(27-77)\end{array}$ \\
\hline & $p$-value * & & \multicolumn{3}{|c|}{0.784} & \multicolumn{3}{|c|}{0.194} & \multicolumn{3}{|c|}{0.169} \\
\hline & & & & & & & & & & & \\
\hline & Invasive ductal carcinoma & $\begin{array}{c}152 \\
(57.8)\end{array}$ & $\begin{array}{c}13 \\
(68.4)\end{array}$ & $\begin{array}{c}2 \\
28.6)\end{array}$ & $\begin{array}{c}137 \\
(57.8)\end{array}$ & $\begin{array}{c}9 \\
90.0)\end{array}$ & $\begin{array}{c}8 \\
(40.0)\end{array}$ & $\begin{array}{c}135 \\
(59.2)\end{array}$ & $\begin{array}{c}21 \\
(63.6)\end{array}$ & $\begin{array}{c}10 \\
(40.0)\end{array}$ & $\begin{array}{c}121 \\
(59.0)\end{array}$ \\
\hline & Invasive lobular carcinoma & $\begin{array}{c}33 \\
(12.6)\end{array}$ & $\begin{array}{c}3 \\
(15.8)\end{array}$ & $\begin{array}{c}1 \\
(14.2)\end{array}$ & $\begin{array}{c}29 \\
(12.2)\end{array}$ & $\begin{array}{c}4 \\
(26.7)\end{array}$ & $\begin{array}{c}1 \\
(5.0)\end{array}$ & $\begin{array}{c}28 \\
(12.3)\end{array}$ & $\begin{array}{c}7 \\
(21.2)\end{array}$ & $\begin{array}{c}1 \\
(4.0)\end{array}$ & $\begin{array}{c}25 \\
(12.2)\end{array}$ \\
\hline & Other invasive hystotypes & $\begin{array}{c}47 \\
(17.8)\end{array}$ & $\begin{array}{c}3 \\
(15.8)\end{array}$ & $\begin{array}{c}2 \\
(28.6)\end{array}$ & $\begin{array}{c}42 \\
(17.8)\end{array}$ & $\begin{array}{c}2 \\
(13.3)\end{array}$ & $\begin{array}{c}5 \\
(25.0)\end{array}$ & $\begin{array}{c}40 \\
(17.5)\end{array}$ & $\begin{array}{c}5 \\
(15.2)\end{array}$ & $\begin{array}{c}6 \\
(24.0)\end{array}$ & $\begin{array}{c}36 \\
(17.6)\end{array}$ \\
\hline & $p$-value * & & \multicolumn{3}{|c|}{0.418} & \multicolumn{3}{|c|}{0.047} & \multicolumn{3}{|c|}{0.005} \\
\hline \multicolumn{12}{|l|}{ Grading } \\
\hline & Well-differentiated & $\begin{array}{c}21 \\
(8.0) \\
\end{array}$ & $\begin{array}{c}4 \\
(21,1)\end{array}$ & $\begin{array}{c}2 \\
(28.6)\end{array}$ & $\begin{array}{c}35 \\
(14.8) \\
\end{array}$ & $\begin{array}{c}2 \\
(13.2)\end{array}$ & $\begin{array}{c}4 \\
(20.0)\end{array}$ & $\begin{array}{c}35 \\
(15.4)\end{array}$ & $\begin{array}{c}6 \\
(18.2) \\
\end{array}$ & $\begin{array}{c}6 \\
(24.0) \\
\end{array}$ & $\begin{array}{c}29 \\
(51.7)\end{array}$ \\
\hline & Moderately differentiated & $\begin{array}{c}100 \\
(38.0)\end{array}$ & $\begin{array}{c}5 \\
(26.3)\end{array}$ & $\begin{array}{c}2 \\
(28.6)\end{array}$ & $\begin{array}{c}93 \\
(39.2)\end{array}$ & $\begin{array}{c}7 \\
7 \\
(46.8)\end{array}$ & $\begin{array}{c}8 \\
(40.0)\end{array}$ & $\begin{array}{c}85 \\
(37.3)\end{array}$ & $\begin{array}{c}12 \\
(36.4)\end{array}$ & $\begin{array}{c}9 \\
96.0)\end{array}$ & $\begin{array}{c}12 \\
(21.4)\end{array}$ \\
\hline & Poorly differentiated & $\begin{array}{c}101 \\
(38.4)\end{array}$ & $\begin{array}{c}10 \\
(52.6)\end{array}$ & $\begin{array}{c}1 \\
(14.2)\end{array}$ & $\begin{array}{c}90 \\
(38.0)\end{array}$ & $\begin{array}{c}6 \\
(40.0)\end{array}$ & $\begin{array}{c}5 \\
(25.0)\end{array}$ & $\begin{array}{c}90 \\
(39.5)\end{array}$ & $\begin{array}{c}15 \\
(45.4)\end{array}$ & $\begin{array}{c}6 \\
(24.0)\end{array}$ & $\begin{array}{c}15 \\
(15.9)\end{array}$ \\
\hline & Missing & $\begin{array}{c}41 \\
(15.6)\end{array}$ & $\begin{array}{c}0 \\
(0.0)\end{array}$ & $\begin{array}{c}2 \\
(28.6)\end{array}$ & $\begin{array}{c}19 \\
(8.0)\end{array}$ & $\begin{array}{c}0 \\
(0.0)\end{array}$ & $\begin{array}{c}3 \\
(15.0)\end{array}$ & $\begin{array}{c}18 \\
(7.8)\end{array}$ & $\begin{array}{c}0 \\
(0.0)\end{array}$ & $\begin{array}{c}4 \\
(16.0)\end{array}$ & $\begin{array}{c}0 \\
(0.0)\end{array}$ \\
\hline & $p$-value * & & \multicolumn{3}{|c|}{0.149} & \multicolumn{3}{|c|}{0.663} & \multicolumn{3}{|c|}{0.232} \\
\hline \multicolumn{12}{|l|}{ Stage } \\
\hline & 0 & $\begin{array}{c}23 \\
(8.8)\end{array}$ & $\begin{array}{c}0 \\
(0.0)\end{array}$ & $\begin{array}{c}0 \\
(0.0)\end{array}$ & $\begin{array}{c}23 \\
(9.7)\end{array}$ & $\begin{array}{c}0 \\
(0.0)\end{array}$ & $\begin{array}{c}4 \\
(20.0)\end{array}$ & $\begin{array}{c}19 \\
(8.3)\end{array}$ & $\begin{array}{c}0 \\
(0.0)\end{array}$ & $\begin{array}{c}4 \\
(16.0\end{array}$ & $\begin{array}{c}19 \\
(9.3)\end{array}$ \\
\hline & I & $\begin{array}{c}104 \\
(39.5)\end{array}$ & $\begin{array}{c}9 \\
(47.4)\end{array}$ & $\begin{array}{c}2 \\
(28.6)\end{array}$ & $\begin{array}{c}93 \\
(39.2)\end{array}$ & $\begin{array}{c}8 \\
(53.4)\end{array}$ & $\begin{array}{c}6 \\
(30.0)\end{array}$ & $\begin{array}{c}90 \\
(39.5)\end{array}$ & $\begin{array}{c}16 \\
(48.5)\end{array}$ & $\begin{array}{c}7 \\
(28.0)\end{array}$ & $\begin{array}{c}81 \\
(39.5)\end{array}$ \\
\hline & II & $\begin{array}{c}65 \\
(24.7)\end{array}$ & $\begin{array}{c}6 \\
(31.6)\end{array}$ & $\begin{array}{c}3 \\
(42.8)\end{array}$ & $\begin{array}{c}56 \\
(23.6)\end{array}$ & $\begin{array}{c}3 \\
(20.0)\end{array}$ & $\begin{array}{c}5 \\
(25.0)\end{array}$ & $\begin{array}{c}57 \\
(25.0)\end{array}$ & $\begin{array}{c}9 \\
(27.2)\end{array}$ & $\begin{array}{c}7 \\
(28.0)\end{array}$ & $\begin{array}{c}49 \\
(23.9)\end{array}$ \\
\hline & III & $\begin{array}{c}28 \\
(10.7)\end{array}$ & $\begin{array}{c}1 \\
(5.2)\end{array}$ & $\begin{array}{c}0 \\
(0.0)\end{array}$ & $\begin{array}{c}27 \\
(11.4)\end{array}$ & $\begin{array}{c}2 \\
(13.3)\end{array}$ & $\begin{array}{c}1 \\
(5.0)\end{array}$ & $\begin{array}{c}25 \\
(11.0)\end{array}$ & $\begin{array}{c}3 \\
(9.1)\end{array}$ & $\begin{array}{c}1 \\
(4.0)\end{array}$ & $\begin{array}{c}24 \\
(11.7)\end{array}$ \\
\hline & IV & $\begin{array}{c}8 \\
(3.0)\end{array}$ & $\begin{array}{c}0 \\
(0.0)\end{array}$ & $\begin{array}{c}0 \\
(0.0)\end{array}$ & $\begin{array}{c}8 \\
(3.4)\end{array}$ & $\begin{array}{c}2 \\
(13.3)\end{array}$ & $\begin{array}{c}0 \\
(0.0)\end{array}$ & $\begin{array}{c}6 \\
(2.6)\end{array}$ & $\begin{array}{c}2 \\
(6.1)\end{array}$ & $\begin{array}{c}0 \\
(0.0)\end{array}$ & $\begin{array}{c}6 \\
(2.9)\end{array}$ \\
\hline
\end{tabular}


Table 3. Cont.

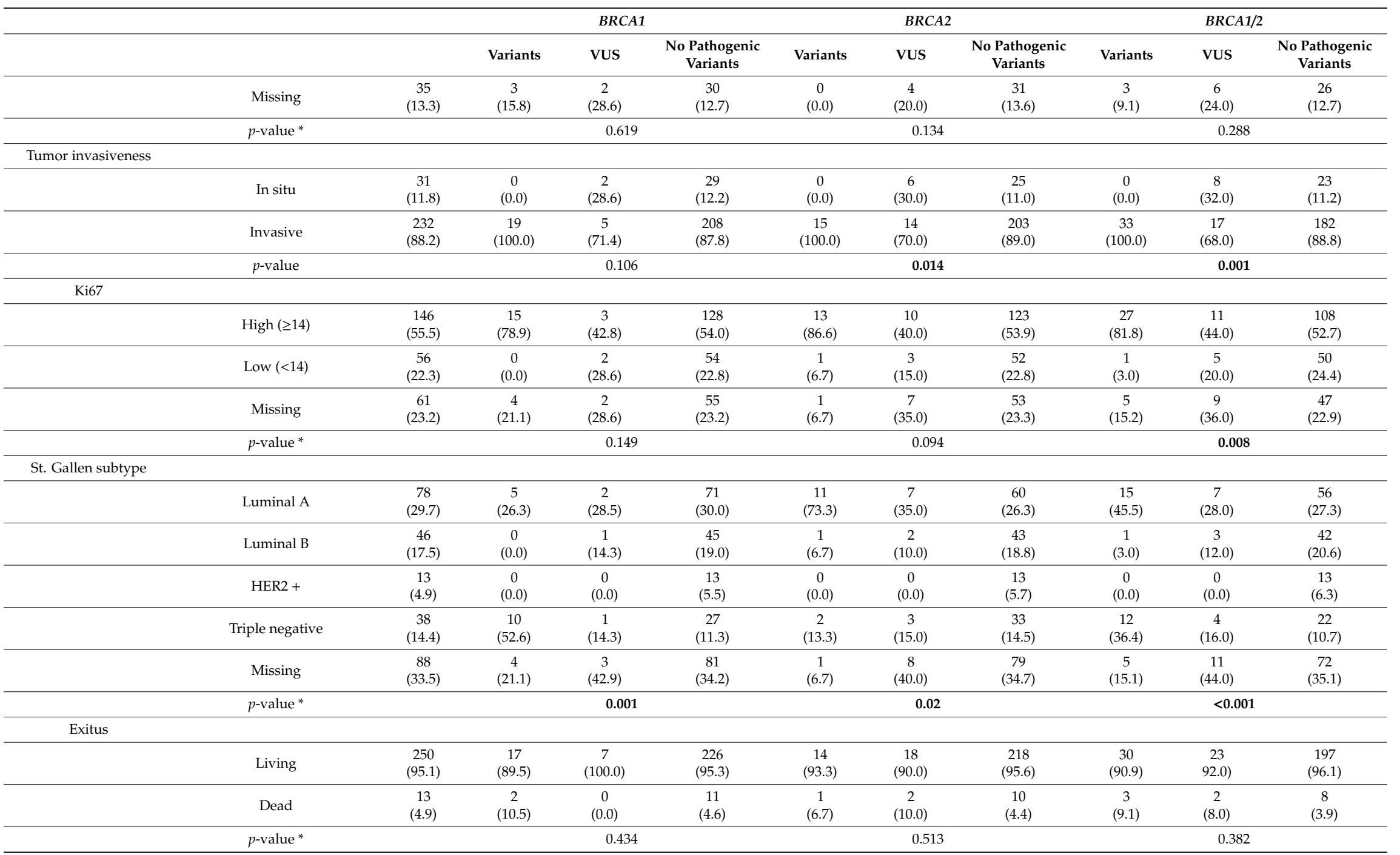

Abbreviations: VUS, Variant of Uncertain Significance; HER2, Human Epidermal Growth Factor Receptor 2. * Pearson Chi-square test or the Fisher Exact test, as appropriate. ** One patients possess the pathogenic variants of the both BRCA1 and BRCA2 genes simultaneously (ID 606). ${ }^{* * *}$ the individuals were all Caucasians. 


\subsection{Characteristics of Breast Cancer in Patients with VUS}

Mean age of the 25 patients with BRCA1/2 VUS was 48 years (range 34-68) and the most frequent histology was ductal (40.0\%), followed by lobular $4.0 \%$ with other invasive hystotypes $24.0 \%$. Grade 1 was detected in $24.0 \%$ of breast cancer, G2 in 36.0\%, G3 in 24.0\%; information about grading was missing in $16.0 \%$ of cases. According to surrogate definitions of intrinsic subtypes of breast cancer [20], $16.0 \%$ of tumors were classified as triple negative, $28.0 \%$ as luminal a-like breast cancer and $12.0 \%$ as luminal b-like. No enriched HER-2 was found in patients with BRCA1/2 VUS. Figure 2 shows the pedigree of a family with VUS. The proband harboring the c.4928T > C variant in the BRCA2 gene was diagnosed with breast cancer at the age of 39; her mother suffered from bilateral BC and carried the same VUS. Her aunt (mother's sister) died of breast cancer as did her grandmother (BRCA test not performed). This VUS seems representative of the hereditary factor of BC due to the frequency of cases with bilateral breast cancer and the onset in youth in three relatives present in the maternal line (mother, aunt and maternal grandmother).
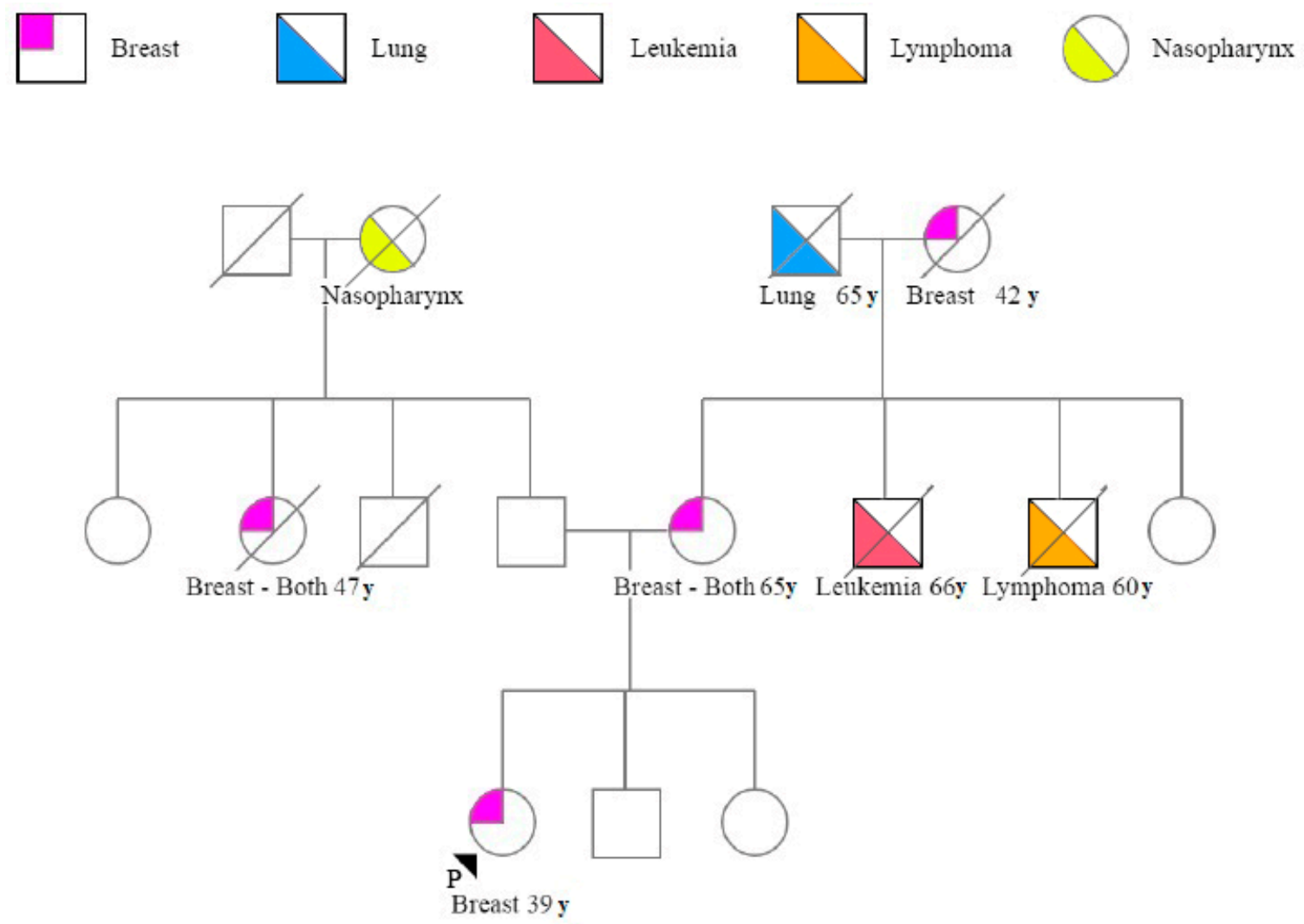

Figure 2. Pedigree of patient ID 886 with c.4928T >C, p.(Val1643Ala) Unclassified variant in BRCA2 gene. The proband is indicated by a black arrow. Cancer Type and age at cancer diagnosis is indicated in the legend. Symbols: squares = males, circles = females; quadrant shading = cancer affected; slash through square or circle $=$ deceased.

\section{Discussion}

This is a Central Italian study evaluating the prevalence and spectrum of $B R C A 1 / 2$ variants. We focused our study on variant detection rates and genetic characteristics associated with specific selection criteria for BRCA1/2 testing in high-risk families and patients affected by breast cancer, whereas other authors evaluated clinical implications and strategy of surveillance of women at high risk. Thirteen percent of the individuals evaluated were carriers of a pathogenic variant, according to the range shown in other countries [27-30], excluding Ashkenazi Jewish ancestry in which founder variants were prevalent [31]. The incidence of $B R C A 1$ and $B R C A 2$ variants was $7.7 \%$ and $6.3 \%$, 
respectively. According to the literature, we report an incidence of TNBC in BRCA-carriers (36.4\%) about 2-fold higher than that found in sporadic breast cancer. TNBC has been reported to account for $12-24 \%$ of all BCs and is associated with an hereditary disease cause [32,33]. Approximately $70 \%$ of $\mathrm{BC}$ s found in $B R C A 1$ variant carriers and up to $23 \%$ of $\mathrm{BCs}$ in $B R C A 2$ carriers are triple-negative [34]. Therefore, according to national and international guidelines, women with TNBC diagnosed at an age $\leq 50-60$ years, irrespective of a positive cancer family history, are eligible for germline $B R C A$ testing [11-13]. As reported in the literature [35,36], BRCA-mutated BC patients showed a significant number of triple-negative cancers $(p<0.001)$ and higher Ki-67 expression $(p=0.008)$ than in other patients (Table 3), which represents the higher aggressiveness of the disease. BRCA1 pathogenic/likely pathogenic variants reported in our study were higher than BRCA2 variants $(54.9 \%$ and $45.0 \%$, respectively). More than 2000 different variants have been identified in BRCA1/2 genes and in some populations, founder variants are the most prevalent ones. For example, up to $2.5 \%$ of the general Ashkenazi Jewish population will harbor variants in BRCA1 c.68_69delAG (also known as 185delAG), c.5266dupC (also known as 5382insC) or BRCA2 c.5946delT (also known as 6174delT) [37].

We observed 30 distinct pathogenic/likely pathogenic BRCA variants (14 in BRCA1 and 16 in $B R C A 2)$ and while 23 were observed only once, 5 in BRCA1 and 2 in BRCA2 variants were detected at least two or more times. These seven variants were detected in $23.3 \%$ of all the patients with pathogenic $B R C A$ variant and almost all of them were observed in exon 20 of $B R C A 1$ and exon 11 of BRCA2. It is important to screen individual populations and ethnic groups to evaluate the true prevalence of $B R C A$ germline variants [38], as the frequency and type of $B R C A$ variants vary significantly depending on ethnicity and race. To our knowledge, our BRCA study on an Italian population (breast/ovarian cancer patients and healthy population) showed that when several recurrent pathogenic variants are detected, these may be considered as founder variants for this population. If confirmed by further studies, this could have significant implications for preventive population screening and targeted treatments with PARP inhibitors. In our cohort, the BRCA1 c.5266dupC (also known as 5382dupC), considered the founder variant of North-Eastern European origin, was the most frequent, representing $23 \%$ of $B R C A 1$ variant carriers, as reported in a previous Italian study [39].

In our study, of the 30 pathogenic-likely pathogenic variants observed, $2(6.6 \%)$ are novel and it will be necessary to evaluate their level of penetration in carrier families.

Moreover, different $B R C A$ variants lead to protein alterations that could have a different impact on the risk of developing tumors in $B R C A$ variant carriers [40].

If a high risk $B R C A$ variant should be detected, it is important to perform genetic counselling to guide patients and their families regarding risk reduction options and treatment. In our study, we have reported a list of the VUS identified (mostly missense variants) and we note a lack of consensus about their biological/clinical significance among the different databases. Based on the frequency or the co-occurrence of pathogenic variants of these VUS, found in the small number of cases tested in our center, it was not possible to classify these variants. Even though clinician's decisions cannot be made based on VUS, some of our findings are worthy of attention and deserve further investigation. This is the case, for example, of the young patient (39 years old) with the variant c.4928T $>$ C reported in BRCA2 (Figure 2). Segregation analysis and functional studies should be further performed in this family due to the absence of consensus among databases. Moreover, other breast/ovarian cancer predisposition genes (already present in commercial panels) should also be investigated by next-generation sequencing.

A strength of our study is that it considers not only the affected individuals but also healthy people considered at risk on the basis of the Cuzick-Tyrer program (life-time risk cut off: $10 \%$ ). Indeed, studies evaluating only patients affected might lead to an overestimate of probability of detecting a variant.

A possible limitation of our study is the selection of individuals for testing. Women should probably not be selected for $B R C A$ testing using only protocols based on risk evaluation tools and strict probability thresholds. Furthermore, there are several different tools to evaluate $B R C A$ risk, and we do not know which is best. Of course, programs with a proactive approach of genetic counseling 
probably need to enforce rigid selection criteria based on probability threshold in order to contain costs and safeguard their feasibility and ethical sustainability. Besides the variant risk, a woman's personal motivation and the potential utility of test results for the family should be considered. Another limitation of our study is the absence of segregation analysis within family members that could facilitate follow up of people at high risk of disease and their relatives.

Notwithstanding these limitations, our study provides the identification of patients with heterozygous variants of both $B R C A 1$ and $B R C A 2$, along with individuals carrying one variant and a VUS, underlining the necessity of complete $B R C A 1 / 2$ testing, which should be offered to all eligible individuals.

The increase of genetic testing leads to the probability of having an non-informative result or VUS. For the management of VUS, it is important to evaluate family history, clinical factors and functional studies on $B R C A$ protein.

Because this information can be confusing and anxiety-provoking to patients, international collaborative efforts are strongly encouraged to ensure that data pertaining to VUS are publicly available.

\section{Conclusions}

Our study reveals that the overall frequency of $B R C A$ germline variants in the selected high-risk central Italian population (BC or OC patients and healthy individuals with elevated risk of hereditary $\mathrm{BC}$ or $\mathrm{OC}$ ) is about $13.8 \%$. Further, several recurrent pathogenic variants detected could be considered as founder variants, if confirmed by further studies. We believe that our results could have significant implications for preventive strategies for unaffected $B R C A$-carriers and effective targeted treatments such as PARP inhibitors for patients with BC or OC.

Author Contributions: Conceived and designed of the study: J.F., V.L., L.P.; involved in the conduct of the study A.M., E.M., P.A., C.M., A.A.-R., F.R.; data interpretation and statistical analysis: F.B., F.S., V.L.; performed sequencing analysis: L.P., M.S.R., F.R.T., V.L.; wrote the paper: J.F., V.L., F.B., L.P., M.S.R. All authors revised and approved the final manuscript.

Funding: This research was supported in part (reagents for $B R C A$ analysis) by the Umbria Association Against Cancer (AUCC).

Acknowledgments: We thank all individuals who participated at the study.

Conflicts of Interest: The authors declare no conflict of interest.

\section{References}

1. Bray, F.; Ferlay, J.; Soerjomataram, I.; Siegel, R.L.; Torre, L.A.; Jemal, A. Global cancer statistics 2018: GLOBOCAN estimates of incidence and mortality worldwide for 36 cancers in 185 countries. CA Cancer J. Clin. 2018, 68, 394-424. [CrossRef] [PubMed]

2. Ferla, R.; Calo, V.; Cascio, S.; Rinaldi, G.; Badalamenti, G.; Carreca, I.; Surmacz, E.; Colucci, G.; Bazan, V.; Russo, A. Founder variants in BRCA1 and BRCA2 genes. Ann. Oncol. 2007, 18, vi93-vi98. [CrossRef] [PubMed]

3. Begg, C.B.; Haile, R.W.; Borg, Å.; Malone, K.E.; Concannon, P.; Thomas, D.C.; Langholz, B.; Bernstein, L.; Olsen, J.H.; Lynch, C.F.; et al. Variation of Breast Cancer Risk Among BRCA1/2 Carriers. JAMA 2008, 299, 194-201. [CrossRef] [PubMed]

4. Wang, F.; Fang, Q.; Ge, Z.; Yu, N.; Xu, S.; Fan, X. Common BRCA1 and BRCA2 variants in breast cancer families: A meta-analysis from systematic review. Mol. Biol. Rep. 2012, 39, 2109-2118. [CrossRef]

5. Judkins, T.; Rosenthal, E.; Arnell, C.; Burbidge, L.A.; Geary, W.; Barrus, T.; Schoenberger, J.; Trost, J.; Wenstrup, R.J.; Roa, B.B. Clinical significance of large rearrangements in BRCA1 and BRCA2. Cancer 2012, 118, 5210-5216. [CrossRef]

6. Kuchenbaecker, K.B.; Hopper, J.L.; Barnes, D.R.; Phillips, K.-A.; Mooij, T.M.; Roos-Blom, M.-J.; Jervis, S.; van Leeuwen, F.E.; Milne, R.L.; Andrieu, N.; et al. Risks of Breast, Ovarian, and Contralateral Breast Cancer for BRCA1 and BRCA2 Variant Carriers. JAMA 2017, 317, 2402. [CrossRef] 
7. Maxwell, K.N.; Wubbenhorst, B.; D’Andrea, K.; Garman, B.; Long, J.M.; Powers, J.; Rathbun, K.; Stopfer, J.E.; Zhu, J.; Bradbury, A.R.; et al. Prevalence of variants in a panel of breast cancer susceptibility genes in BRCA1/2-negative patients with early-onset breast cancer. Genet. Med. 2015, 17, 630-638. [CrossRef]

8. Schon, K.; Tischkowitz, M. Clinical implications of germline variants in breast cancer: TP53. Breast Cancer Res. Treat. 2018, 167, 417-423. [CrossRef]

9. Pinto, C.; Mangone, L. Epidemiology of cancer in Italy: From real data to the need for cancer networks. Recenti Prog. Med. 2016, 107, 505-506. [CrossRef]

10. Gradishar, W.J.; Anderson, B.O.; Balassanian, R.; Blair, S.L.; Burstein, H.J.; Cyr, A.; Elias, A.D.; Farrar, W.B.; Forero, A.; Giordano, S.H.; et al. Breast Cancer, Version 4.2017, NCCN Clinical Practice Guidelines in Oncology. J. Natl. Compr. Cancer Netw. 2018, 16, 310-320. [CrossRef]

11. Paluch-Shimon, S.; Cardoso, F.; Sessa, C.; Balmana, J.; Cardoso, M.J.; Gilbert, F.; Senkus, E. ESMO Guidelines Committee Prevention and screening in $B R C A$ variant carriers and other breast/ovarian hereditary cancer syndromes: ESMO Clinical Practice Guidelines for cancer prevention and screening. Ann. Oncol. 2016, 27, v103-v110. [CrossRef] [PubMed]

12. Pinto, C.; Bella, M.A.; Capoluongo, E.; Carrera, P.; Clemente, C.; Colombo, N.; Cortesi, L.; De Rosa, G.; Fenizia, F.; Genuardi, M.; et al. Recommendations for the implementation of BRCA testing in the care and treatment pathways of ovarian cancer patients. Futur. Oncol. 2016, 12, 2071-2075. [CrossRef] [PubMed]

13. Underhill, C.; Toulmonde, M.; Bonnefoi, H. A review of PARP inhibitors: From bench to bedside. Ann. Oncol. 2011, 22, 268-279. [CrossRef]

14. Linee Guida Neoplasie Della Mammella | AIOM. Available online: https://www.aiom.it/linee-guida-aiom2018-neoplasie-della-mammella-11/ (accessed on 6 June 2019).

15. Antoniou, A.C.; Easton, D.F. Models of genetic susceptibility to breast cancer. Oncogene 2006, 25, 5898-5905. [CrossRef] [PubMed]

16. BRCAPRO | BayesMendel Lab. Available online: https://projects.iq.harvard.edu/bayesmendel/BRCApro (accessed on 6 June 2019).

17. Parmigiani, G.; Berry, D.; Aguilar, O. Determining carrier probabilities for breast cancer-susceptibility genes BRCA1 and BRCA2. Am. J. Hum. Genet. 1998, 62, 145-158. [CrossRef] [PubMed]

18. Tyrer, J.; Duffy, S.W.; Cuzick, J. A breast cancer prediction model incorporating familial and personal risk factors. Stat. Med. 2004, 23, 1111-1130. [CrossRef]

19. Goldhirsch, A.; Winer, E.P.; Coates, A.S.; Gelber, R.D.; Piccart-Gebhart, M.; Thürlimann, B.; Senn, H.-J.; Albain, K.S.; André, F.; Bergh, J.; et al. Personalizing the treatment of women with early breast cancer: Highlights of the St Gallen International Expert Consensus on the Primary Therapy of Early Breast Cancer 2013. Ann. Oncol. 2013, 24, 2206-2223. [CrossRef]

20. Plon, S.E.; Eccles, D.M.; Easton, D.; Foulkes, W.D.; Genuardi, M.; Greenblatt, M.S.; Hogervorst, F.B.L.; Hoogerbrugge, N.; Spurdle, A.B.; Tavtigian, S.V.; et al. Sequence variant classification and reporting: Recommendations for improving the interpretation of cancer susceptibility genetic test results. Hum. Mutat. 2008, 29, 1282-1291. [CrossRef]

21. Szabo, C.; Masiello, A.; Ryan, J.F.; Brody, L.C. The Breast Cancer Information Core: Database design, structure, and scope. Hum. Mutat. 2000, 16, 123-131. [CrossRef]

22. Caputo, S.; Benboudjema, L.; Sinilnikova, O.; Rouleau, E.; Béroud, C.; Lidereau, R. French BRCA GGC Consortium Description and analysis of genetic variants in French hereditary breast and ovarian cancer families recorded in the UMD-BRCA1/BRCA2 databases. Nucleic Acids Res. 2012, 40, D992-D1002. [CrossRef]

23. Fokkema, I.F.A.C.; Taschner, P.E.M.; Schaafsma, G.C.P.; Celli, J.; Laros, J.F.J.; den Dunnen, J.T. LOVD v.2.0: The next generation in gene variant databases. Hum. Mutat. 2011, 32, 557-563. [CrossRef] [PubMed]

24. Richards, S.; Aziz, N.; Bale, S.; Bick, D.; Das, S.; Gastier-Foster, J.; Grody, W.W.; Hegde, M.; Lyon, E.; Spector, E.; et al. Standards and guidelines for the interpretation of sequence variants: A joint consensus recommendation of the American College of Medical Genetics and Genomics and the Association for Molecular Pathology. Genet. Med. 2015, 17, 405-423. [CrossRef] [PubMed]

25. den Dunnen, J.T.; Dalgleish, R.; Maglott, D.R.; Hart, R.K.; Greenblatt, M.S.; McGowan-Jordan, J.; Roux, A.-F.; Smith, T.; Antonarakis, S.E.; Taschner, P.E.M.; et al. On behalf of the Human Genome Variation Society (HGVS), the Human Variome Project (HVP), and the Human Genome Organisation (HUGO):HGVS Recommendations for the Description of Sequence Variants: 2016 Update. Hum. Mutat. 2016, 6, 564-569. [CrossRef] 
26. Bianconi, F.; Brunori, V.; Valigi, P.; La Rosa, F.; Stracci, F. Information technology as tools for cancer registry and regional cancer network integration. IEEE Trans. Syst. Man Cybern. Part A Syst. Hum. 2012, 42, 1410-1424. [CrossRef]

27. Kim, Y.C.; Zhao, L.; Zhang, H.; Huang, Y.; Cui, J.; Xiao, F.; Downs, B.; Wang, S.M. Prevalence and spectrum of $B R C A$ germline variants in mainland Chinese familial breast and ovarian cancer patients. Oncotarget 2016, 7, 9600-9612. [CrossRef] [PubMed]

28. Alvarez, C.; Tapia, T.; Perez-Moreno, E.; Gajardo-Meneses, P.; Ruiz, C.; Rios, M.; Missarelli, C.; Silva, M.; Cruz, A.; Matamala, L.; et al. BRCA1 and BRCA2 founder variants account for $78 \%$ of germline carriers among hereditary breast cancer families in Chile. Oncotarget 2017, 8, 74233-74243. [CrossRef]

29. Sung, P.L.; Wen, K.C.; Chen, Y.J.; Chao, T.C.; Tsai, Y.F.; Tseng, L.M.; Qiu, J.T.T.; Chao, K.C.; Wu, H.H.; Chuang, C.M.; et al. The frequency of cancer predisposition gene variants in hereditary breast and ovarian cancer patients in Taiwan: From BRCA1/2 to multi-gene panels. PLoS ONE 2017, 12, e0185615. [CrossRef]

30. Loizidou, M.A.; Hadjisavvas, A.; Pirpa, P.; Spanou, E.; Delikurt, T.; Tanteles, G.A.; Daniel, M.; Kountourakis, P.; Malas, S.; Ioannidis, G.; et al. BRCA1 and BRCA2 variant testing in Cyprus; a population based study. Clin. Genet. 2017, 91, 611-615. [CrossRef]

31. Szabo, C.I.; King, M.C. Population genetics of BRCA1 and BRCA2. Am. J. Hum. Genet. 1997, 60, $1013-1020$.

32. Foulkes, W.D.; Smith, I.E.; Reis-Filho, J.S. Triple-Negative Breast Cancer. N. Engl. J. Med. 2010, 363, $1938-1948$. [CrossRef]

33. Metcalfe, K.; Lubinski, J.; Lynch, H.T.; Ghadirian, P.; Foulkes, W.D.; Kim-Sing, C.; Neuhausen, S.; Tung, N.; Rosen, B.; Gronwald, J.; et al. Family History of Cancer and Cancer Risks in Women with BRCA1 or BRCA2 Variants. J. Natl. Cancer Inst. 2010, 102, 1874-1878. [CrossRef]

34. Stevens, K.N.; Vachon, C.M.; Couch, F.J. Genetic Susceptibility to Triple-Negative Breast Cancer. Cancer Res. 2013, 73, 2025-2030. [CrossRef] [PubMed]

35. Templeton, A.J.; Gonzalez, L.D.; Vera-Badillo, F.E.; Tibau, A.; Goldstein, R.; Šeruga, B.; Srikanthan, A.; Pandiella, A.; Amir, E.; Ocana, A. Interaction between Hormonal Receptor Status, Age and Survival in Patients with BRCA1/2 Germline Variants: A Systematic Review and Meta-Regression. PLoS ONE 2016, 11, e0154789. [CrossRef] [PubMed]

36. Wang, W.; Wu, J.; Zhang, P.; Fei, X.; Zong, Y.; Chen, X.; Huang, O.; He, J.-R.; Chen, W.; Li, Y.; et al. Prognostic and predictive value of Ki-67 in triple-negative breast cancer. Oncotarget 2016, 7, 31079-31087. [CrossRef]

37. Roa, B.B.; Boyd, A.A.; Volcik, K.; Richards, C.S. Ashkenazi Jewish population frequencies for common variants in BRCA1 and BRCA2. Nat. Genet. 1996, 14, 185-187. [CrossRef] [PubMed]

38. Fackenthal, J.D.; Olopade, O.I. Breast cancer risk associated with BRCA1 and BRCA2 in diverse populations. Nat. Rev. Cancer 2007, 7, 937-948. [CrossRef]

39. Azzollini, J.; Scuvera, G.; Bruno, E.; Pasanisi, P.; Zaffaroni, D.; Calvello, M.; Pasini, B.; Ripamonti, C.B.; Colombo, M.; Pensotti, V.; et al. Variant detection rates associated with specific selection criteria for BRCA1/2 testing in 1854 high-risk families: A monocentric Italian study. Eur. J. Intern. Med. 2016, 32, 65-71. [CrossRef]

40. Finkelman, B.S.; Rubinstein, W.S.; Friedman, S.; Friebel, T.M.; Dubitsky, S.; Schonberger, N.S.; Shoretz, R.; Singer, C.F.; Blum, J.L.; Tung, N.; et al. Breast and ovarian cancer risk and risk reduction in Jewish BRCA1/2 variant carriers. J. Clin. Oncol. 2012, 30, 1321-1328. [CrossRef]

(C) 2020 by the authors. Licensee MDPI, Basel, Switzerland. This article is an open access article distributed under the terms and conditions of the Creative Commons Attribution (CC BY) license (http://creativecommons.org/licenses/by/4.0/). 